\title{
EchoGéo
}

$51 \mid 2020$

Territoires « cyclonés "

\section{Les temps de la catastrophe}

L'ouragan de septembre 1928 en Guadeloupe

Jérémy Desarthe

\section{CpenEdition}

Journals

Édition électronique

URL : https://journals.openedition.org/echogeo/19116

DOI : 10.4000/echogeo.19116

ISSN : 1963-1197

Éditeur

Pôle de recherche pour l'organisation et la diffusion de l'information géographique (CNRS UMR 8586)

Référence électronique

Jérémy Desarthe, «Les temps de la catastrophe », EchoGéo [En ligne], 51 | 2020, mis en ligne le 25 avril 2020, consulté le 10 août 2021. URL : http://journals.openedition.org/echogeo/19116; DOI : https://doi.org/10.4000/echogeo.19116

Ce document a été généré automatiquement le 10 août 2021.

EchoGéo est mis à disposition selon les termes de la licence Creative Commons Attribution - Pas d'Utilisation Commerciale - Pas de Modification 4.0 International (CC BY-NC-ND) 


\title{
Les temps de la catastrophe
}

\author{
L'ouragan de septembre 1928 en Guadeloupe
}

\author{
Jérémy Desarthe
}

1 En frappant les Antilles dans la nuit du 5 au 6 septembre 2017, l'ouragan Irma est venu rappeler l'exposition des îles-du-Nord aux risques cycloniques. Deux ans après son passage, l'île de Saint-Martin a amorcé sa reconstruction mais conserve encore les stigmates du passage de l'ouragan pour une durée encore difficile à évaluer. Au regard de l'expérience historique, il est intéressant de s'interroger sur la manière dont les sociétés ont fait face par le passé à de tels événements et selon quelles temporalités. Le cyclone de septembre 1928 constitue avec Irma, Hugo et José les principaux ouragans qui ont touché les Antilles au cours du $\mathrm{XX}^{\mathrm{e}}$ siècle et représente un cas d'étude intéressant (Desarthe et Moncoulon, 2017). Ce cyclone a touché la Guadeloupe et dans une moindre mesure la Martinique entre le 12 et le 13 septembre 1928. Non nommé, puisque cette pratique ne se fera qu'à partir des années 1950, il est connu aux ÉtatsUnis sous le nom Okeechobee en raison des inondations du lac du même nom en Floride. À Porto-Rico, sa mémoire est conservée sous le nom de San Felipe. En Guadeloupe, où le cyclone a été à l'origine de 1120 à 1200 morts, sa mémoire est encore vive et donne lieu à des commémorations régulières.

2 La connaissance des événements passés est incontournable pour appréhender l'exposition des territoires et des sociétés, et pour la construction des politiques de prévention. Dans cette perspective, la Caisse Centrale de Réassurance, en tant qu'acteur $\mathrm{du}$ régime d'indemnisation des Catastrophes naturelles et comme gestionnaire comptable et financier du Fonds de Prévention des Risques Naturels Majeurs, mène depuis plusieurs années des travaux de recherche et de développement intégrant une dimension historique (Desarthe, 2014, 2017, 2019). Les résultats de l'étude menée entre 2013 et 2017 consacrée aux outre-mer sont partagés dans le cadre du projet d'ANR TIREX qui vise à faire un retour d'expérience sur l'ouragan Irma partagé avec les populations afin d'améliorer la résilience des sociétés.

3 L'intérêt des historiens pour l'étude des catastrophes passées est ancien et les travaux ont toujours eu pour ambition d'améliorer la connaissance des sociétés et des aléas (Le Roy Ladurie, 1967 ; Favier 2000, 2002 ; Coeur, 2006 ; Garnier, 2009). Dans les Antilles, 
plusieurs travaux réalisés par des géographes et des historiens (Saffache, 2003 ; Zahibo, 2007 ; Desarthe, 2017) ont permis de proposer des reconstructions chronologiques des cyclones. Au-delà du simple inventaire, l'analyse des événements passés permet également d'appréhender le système risque dans sa dimension spatiale et temporelle (Giacona et al., 2019). Ce faisant, à partir de l'exemple du cyclone de 1928, il s'agit d'analyser la manière dont un phénomène naturel est susceptible d'altérer le fonctionnement d'une société et les réponses apportées par celle-ci. Il s'agit d'aborder la vulnérabilité sous l'angle de la propension des enjeux à subir des dommages (D'Ercole, 1994 ; Veyret, 2007) et à travers l'analyse du chaînage des impacts (Provitolo, 2005 ; Duvat, 2017). La démarche mise en œuvre vise aussi à intégrer les différentes temporalités de la catastrophe afin d'évaluer la résilience de la société guadeloupéenne au lendemain du cyclone de 1928. Définie comme la capacité d'un système à faire face aux conséquences d'un aléa pour restaurer ses structures et ses fonctions essentielles (Walker et al., 2004), elle intègre également une dimension temporelle puisqu'elle mesure en effet la durée nécessaire au retour à un équilibre après une perturbation. Cette période étant fonction de l'ampleur de l'événement, de l'adaptabilité de la société (Dauphine et Provitolo, 2007). La conceptualisation de la vulnérabilité et de la résilience est relativement récente mais ils constituent des outils importants pour interroger le passé (Meschinet de Richemond, 2016).

4 Cette approche est rendue possible grâce à une exploitation et une analyse critique des archives émanant des autorités administratives (Garnier, 2009 ; Desarthe, 2013, 2019). Les pratiques mises en œuvre par le passé au lendemain d'une catastrophe ne sont pas si éloignées de ce que nous connaissons aujourd'hui (Desarthe, 2019). Les autorités cherchent rapidement à dresser un inventaire précis des dommages afin de mettre en œuvre les réponses adaptées à la situation.

5 Ce faisant, les documents conservés aux archives départementales de Guadeloupe et aux archives nationales de l'outre-mer à Aix-en-Provence sur l'ouragan de 1928 contiennent des informations qui permettent d'appréhender tous les aspects de la catastrophe. Ainsi, l'historien dispose d'une riche correspondance entre les différents acteurs (sinistrés, maires, gouverneur, ministre des colonies), d'inventaires de dommages ou encore du détail des mesures prises pour secourir les sinistrés. Au total, pour le cyclone de 1928, ce sont plus d'une centaine de documents de natures variées qui ont été consultés. L'ensemble des données a été regroupé au sein d'une base de données dont le géoréférencement a rendu possible l'exploitation de la dimension spatiale des informations collectées.

$6 \mathrm{Au}$ moment du passage du cyclone, les 240000 habitants qui peuplent l'île vivent essentiellement de l'agriculture, et l'industrie sucrière constitue un facteur de rayonnement économique (Schnakenbourg, 2009). À partir des données collectées, il est possible de s'interroger sur la manière dont les populations et les activités ont été touchées et sur les conséquences de l'événement sur le long terme. L'État, comme au lendemain de chaque catastrophe, est intervenu pour secourir les sinistrés (Favier, 2000 ; Garnier, 2009 ; Desarthe, 2013). Il s'agit alors d'analyser les moyens mis en œuvre et les motivations pour répondre aux besoins des habitants au lendemain du cyclone. L'insularité de l'île et son statut de colonie dans un contexte d'une volonté d'assimilation des populations conduisent à nous interroger sur les modalités de mise en place de ces secours. Cependant, dans un premier temps, il convient de revenir sur 
le phénomène lui-même, sur ses caractéristiques et le contexte météo-cyclonique dans lequel il a touché la Guadeloupe afin de souligner son exceptionnalité.

\section{Chronique d'une catastrophe}

\section{Un événement sans précédent?}

Les cyclones sont avec les séismes, les principaux risques naturels auxquels sont exposés les Antilles françaises. La reconstruction de la sévérité des cyclones pour les Antilles françaises selon la classification Saffir-Simpson menée à partir d'une étude de leurs dommages (Desarthe et Moncoulon, 2017) fournit de précieuses informations sur le contexte météo-cyclonique dans lequel le cyclone de 1928 est intervenu (illustration 1). Le dernier événement qui avait touché la Guadeloupe avait eu lieu le 21 septembre 1908. Il faut remonter à l'ouragan de catégorie 2 survenu le 7 août 1899 pour retrouver trace de dommages importants pour la Guadeloupe. Toutefois, pour retrouver un événement similaire à celui de 1928, il faut remonter au 6 septembre 1865 . À cette époque, la perte des récoltes et la destruction des lieux de production avaient ralenti l'industrie sucrière alors en plein essor (Schnakenbourg, 2000). Cet événement est longtemps resté dans la mémoire des guadeloupéens en raison des nombreux décès causés par le cyclone et par l'épidémie de choléra qui s'était déclarée peu après. Ainsi, la faible occurrence d'événements majeurs durant cet intervalle de 64 années a pu altérer la mémoire et la représentation du risque en accentuant ainsi la vulnérabilité des sociétés (Desarthe, 2013).

Illustration 1 - Les cyclones dans les Antilles françaises depuis 1635

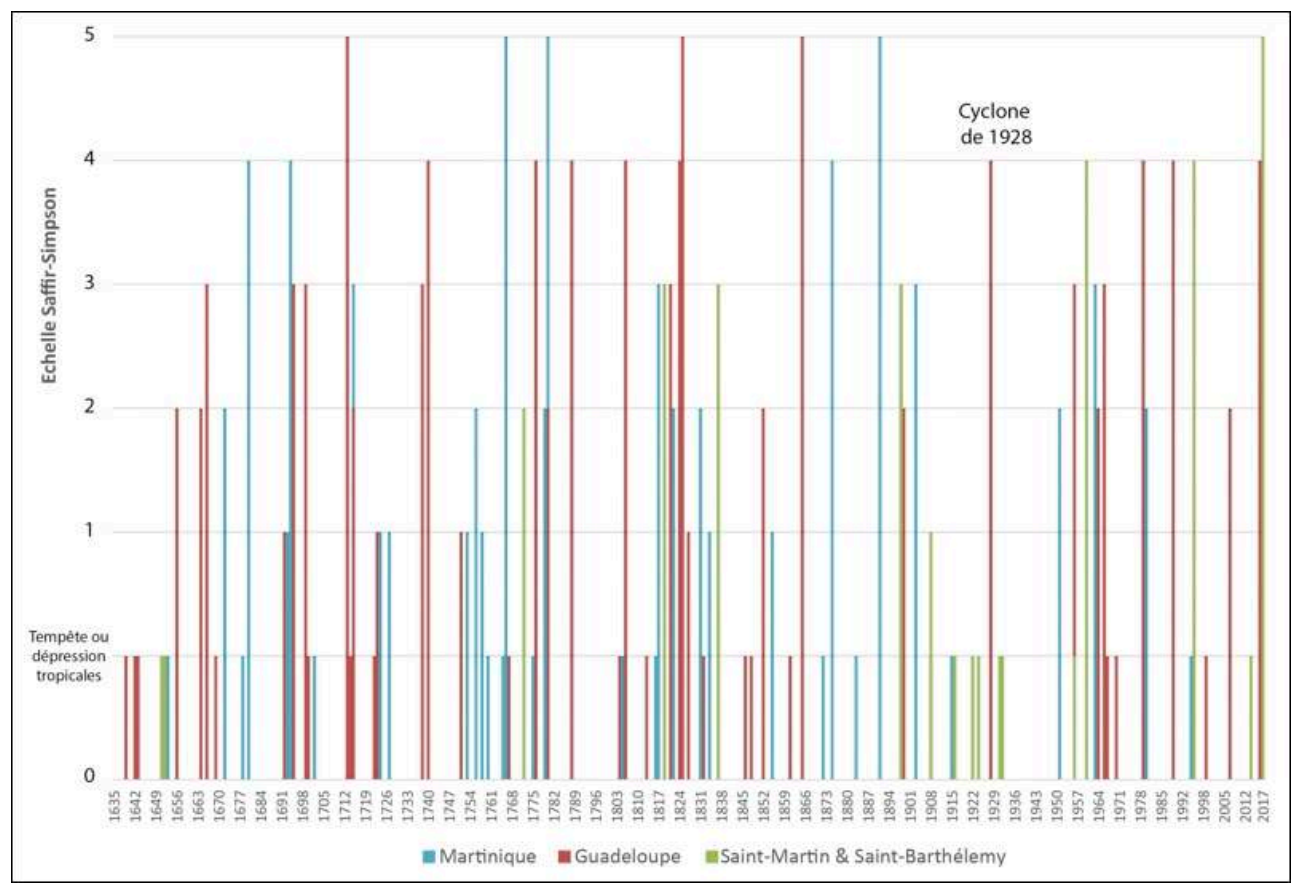

Source : d'après Desarthe J. et Moncoulon D., 2017.

Intéressons-nous maintenant à la saison cyclonique 1928. Débutée en juillet et s'étalant jusqu'en novembre, elle a été relativement calme avec 6 systèmes dépressionnaires 
recensés par la NOAA survenus entre le 3 août et le 15 octobre 1928 contre une dizaine en moyenne chaque année pour la période 1851-2012 selon la base de données HURDAT de la NOAA. Seulement 4 d'entre eux ont atteint le stade de cyclone et un seul - celui du 12 septembre - est classé comme un cyclone majeur (catégorie supérieure à 3 sur la classe Saffir-Simpson). Le premier phénomène est relativement tardif puisqu'il ne se manifeste que le 3 août avec une trajectoire similaire à celle du cyclone qui touche la Guadeloupe le 12 septembre 1928. Le second passe au sud-ouest de l'Arc Antillais épargnant les territoires français des Antilles. La trajectoire des trois autres est assez éloignée des Antilles pour ne pas être à l'origine de dommages (illustration 2).

Illustration 2 - Saison cyclonique et trajectoire de l'ouragan Okeechobee en 1928

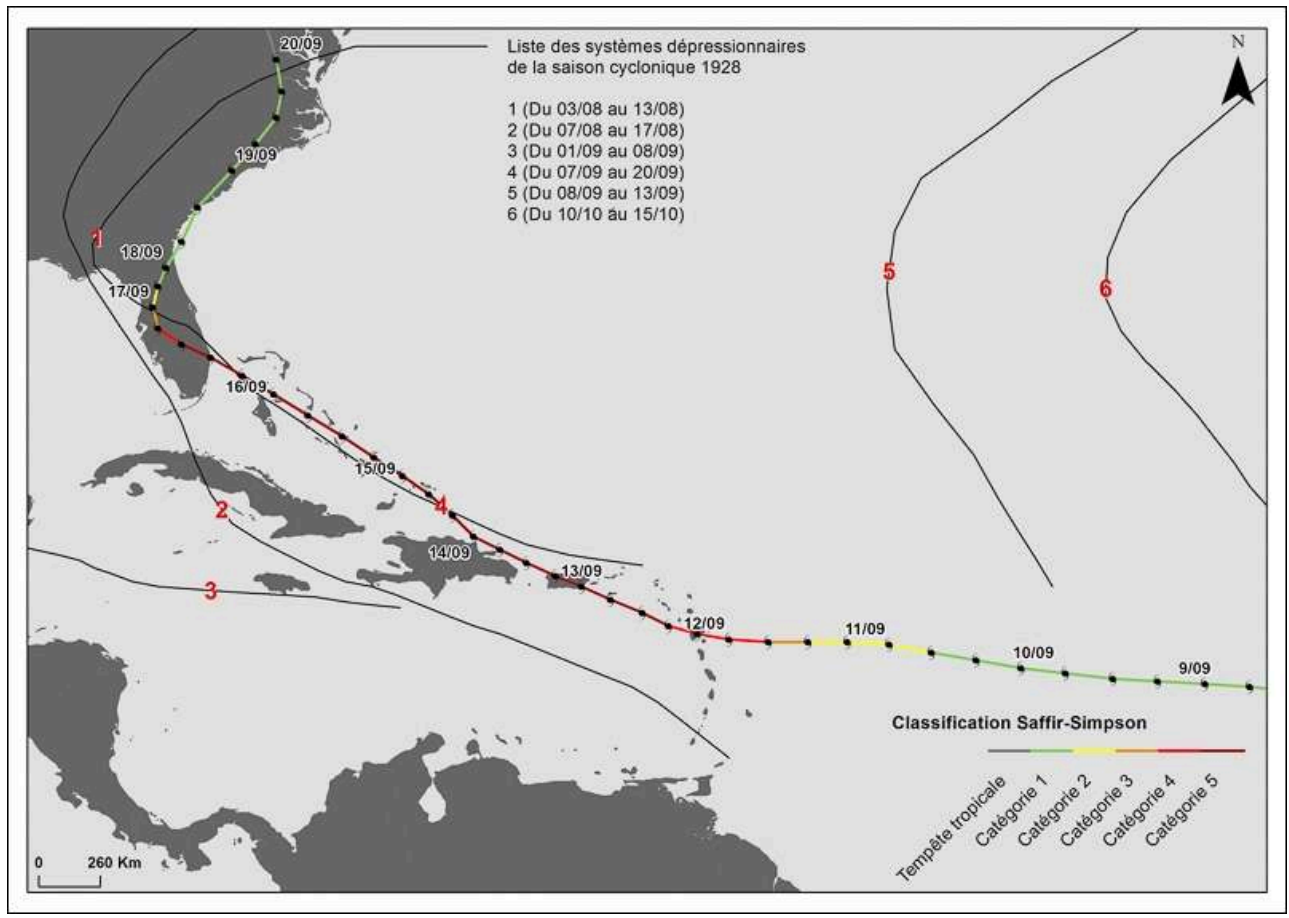

Source : base de données IBTrACS, Hurdat 2, NOAA

\section{Une catastrophe à l'échelle régionale}

Le 11 septembre à $16 \mathrm{~h}$, le gouverneur de la Guadeloupe est averti par télégramme de la présence d'un ouragan à moins de $500 \mathrm{~km}$ de l'île. Il s'agit du premier niveau d'alerte du système de prévision mis en place au début du siècle (Desarthe, 2014). S'appuyant sur un réseau d'observatoires communiquant entre eux grâce au télégraphe, ce système informe les différents territoires en fonction de la menace plusieurs heures avant l'événement. Dans sa correspondance avec le ministre des Colonies, le gouverneur de Guadeloupe explique que le cyclone paraissant devoir être d'une violence extrême. L'examen de la carte fit alors prévoir qu'il allait passer sur la colonie, et l'initiative est prise de prévenir les populations sans attendre l'avis $n^{\circ} 2$ pour annoncer l'imminence $\mathrm{du}$ cyclone comme il est prévu normalement (ANOM, Guadeloupe, carton 244, dossier 1482). Toutes les mesures semblent alors prises pour alerter les populations. Le révérend père Quentin confirme dans son journal que le tambour de la ville de Pointeà-Pitre a annoncé l'arrivée du cyclone peu de temps après (Fabre et Stehle, 1992). 
Parallèlement, le gouverneur demande au service du port d'alerter les compagnies de navigation.

Les premières manifestations du cyclone se font le 12 septembre vers $8 \mathrm{~h}$ du matin (heure locale) avec des vents d'environ $40 \mathrm{~km} / \mathrm{h}$ (ANOM, carton 244, dossier 1482). À $10 \mathrm{~h}$, une baisse importante du baromètre est observée ainsi que la hausse du niveau de la mer. À $11 \mathrm{~h}$, la vitesse des vents se renforce. La toiture du palais de justice constituée de tôles est rapidement emportée. Vers $12 \mathrm{~h}$, la pluie commence à tomber avec violence inondant les habitations dont la couverture a été détruite par le vent. Vers 14h15, la pression atmosphérique atteint son minimum avec $937 \mathrm{hPa}$ au moment du passage de l'œil du cyclone sur la Guadeloupe (Mitchell, 1928). Comme le raconte le révérend père Quentin, c'est à ce moment-là qu'une accalmie se produisit brusquement. "La pluie cessa, le vent aussi [...] Hélas! Cela dura quelques minutes [...] Le ciel s'assombrit de nouveau et le vent reparut aussi violent " (Fabre et Stehle, 1992). Vers 15h, la mer envahit la ville de Pointe-à-Pitre sous l'effet de la surcote atmosphérique et de la violence des vents. L'eau balaye tout sur son passage et transporte de nombreuses barques et bateaux loin à l'intérieur des terres. Les infrastructures portuaires sont détruites et de nombreuses personnes résidantes sur les îlets sont emportées par les eaux. Le vent continu de souffler jusque tard dans la nuit et ce n'est qu'au matin du 13 septembre que celui-ci cesse.

11 Après son passage, le cyclone gagne en intensité pour atteindre la classe 5 et passe sur Porto-Rico le 13 septembre, les Bahamas le 15 et touche la Floride le 16 septembre. Sur son passage, le cyclone détruit une grande partie des infrastructures et des habitations. Dans l'ensemble du bassin caribéen, les dommages sont considérables. À Porto-Rico, le bilan humain fait état de 300 morts et les dommages sont estimés à 50 millions de dollars (Fassig, 1928). Si la destruction des principaux instruments de mesures situés à Basse-Terre et à Pointe-à-Pitre ne permet pas d'avoir de données précises sur la vitesse maximale atteinte par le vent, des estimations a posteriori évoquent environ $220 \mathrm{~km} / \mathrm{h}$ pour Pointe-à-Pitre (ANOM, Guadeloupe, Travaux Publics, carton 1157, dossier 3).

\section{Les dommages causés par le cyclone}

12 Le cyclone laisse derrière lui de nombreux dommages et une population désemparée. La pluie qui tombe les jours suivants exacerbe un peu plus les difficultés. À partir des données collectées, il est possible de préciser la nature des dommages. Au total, les pertes consécutives au passage du cyclone sont évaluées à plus de 800 millions de Francs de l'époque et se répartissent pour 250 millions de francs de dommages aux populations, pour 500 millions de francs pour les pertes agricoles et économiques. Les 40 millions restants concernent les dommages aux infrastructures de l'île (bâtiment publics, routes, ponts).

\section{Les dommages aux particuliers}

"Vous devinez le spectacle qui s'offrit à nos regards quand le jour fut venu (le 13 septembre 1928): on aurait dit une ville qui venait de subir un bombardement [...] Certaines rues étaient complètement barrées par des maisons écroulées, déplacées ou renversées » (Fabre et Stehle, 1992). Les photographies prisent après la catastrophe 
viennent illustrer les propos du révérend père Quentin et ne laissent aucun doute sur l'étendue des dommages (illustration 3) (ANOM, GUA, carton 246, dossier 1492).

Illustration 3 - Vue du faubourg Frébault à Pointe-à-Pitre après le passage du cyclone

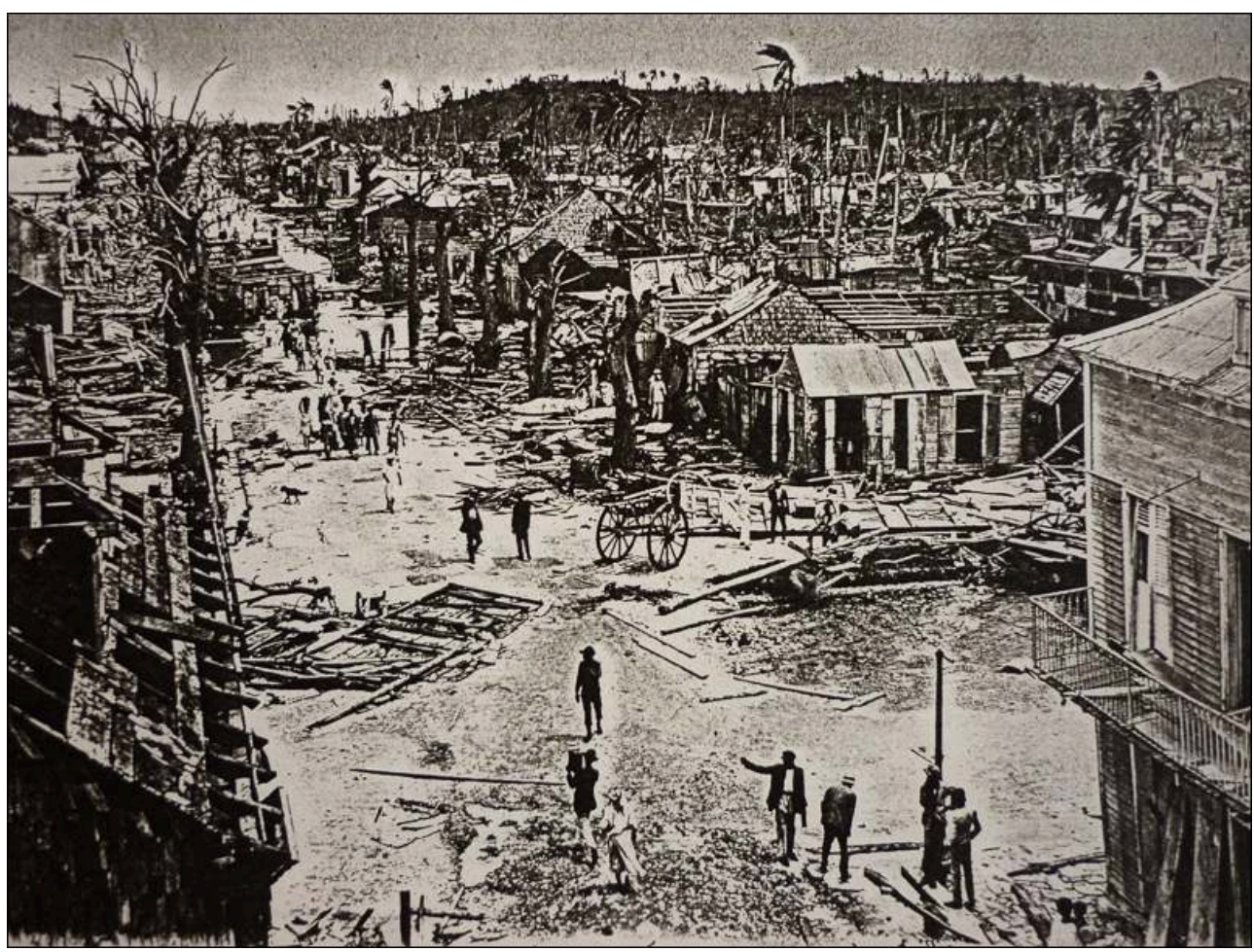

Source : Archives Nationales de l'Outre-Mer, GUA, carton 246, dossier 1492.

14 Avec environ 240000 habitants dont près de la moitié a été touchée par le cyclone, la Guadeloupe se retrouve dans une situation difficile. Après cinq jours sans communication et sans aide de l'extérieur l'arrivée du premier bateau en provenance de la Martinique a permis d'acheminer des vivres et des médicaments et ainsi d'éviter l'émergence de maladies. Durant cette période de nombreuses scènes de pillages ont eu lieu obligeant le gouverneur à promulguer un couvre-feu.

15 L'inventaire réalisé par les services du gouvernement à partir des 34000 déclarations de sinistres reçues totalise pour plus de 250 millions de francs de dommages. À titre de comparaison, les dommages en Martinique s'élèvent à un peu moins de 2000000 francs pour 797 déclarations de sinistres. 628 déclarants de Guadeloupe ont des pertes individuelles supérieures à 30000 francs pour un total de 100 millions de francs soit $40 \%$ du total des dommages. Il n'est toutefois pas possible de dresser un panorama plus précis en l'absence d'information sur le type et la nature des dommages. La case créole constitue le type d'habitation le plus répandu et dans son récit sur les quelques détails sur le cyclone du 12 septembre 1928, le révérend père Quentin signale que dans les faubourgs de Pointe-à-Pitre, l'habitat était en grande partie composé de cases en bois et posées à même le sol. Les descriptions de dommages mentionnent aussi l'usage de tôles comme principaux matériaux de construction pour les toitures qui offrent une faible résistance face aux vents cycloniques. Seul le rapport publié en novembre 1928 précise que plus de 6000 maisons ont été entièrement détruites (ANOM, Carton 244, Dossier 
1486). Cependant, la répartition du montant des dommages permet d'apprécier les communes ayant été le plus touchées (illustration 4).

Illustration 4 - Total des pertes déclarées par les sinistrés de l'ouragan du 12/09/1928

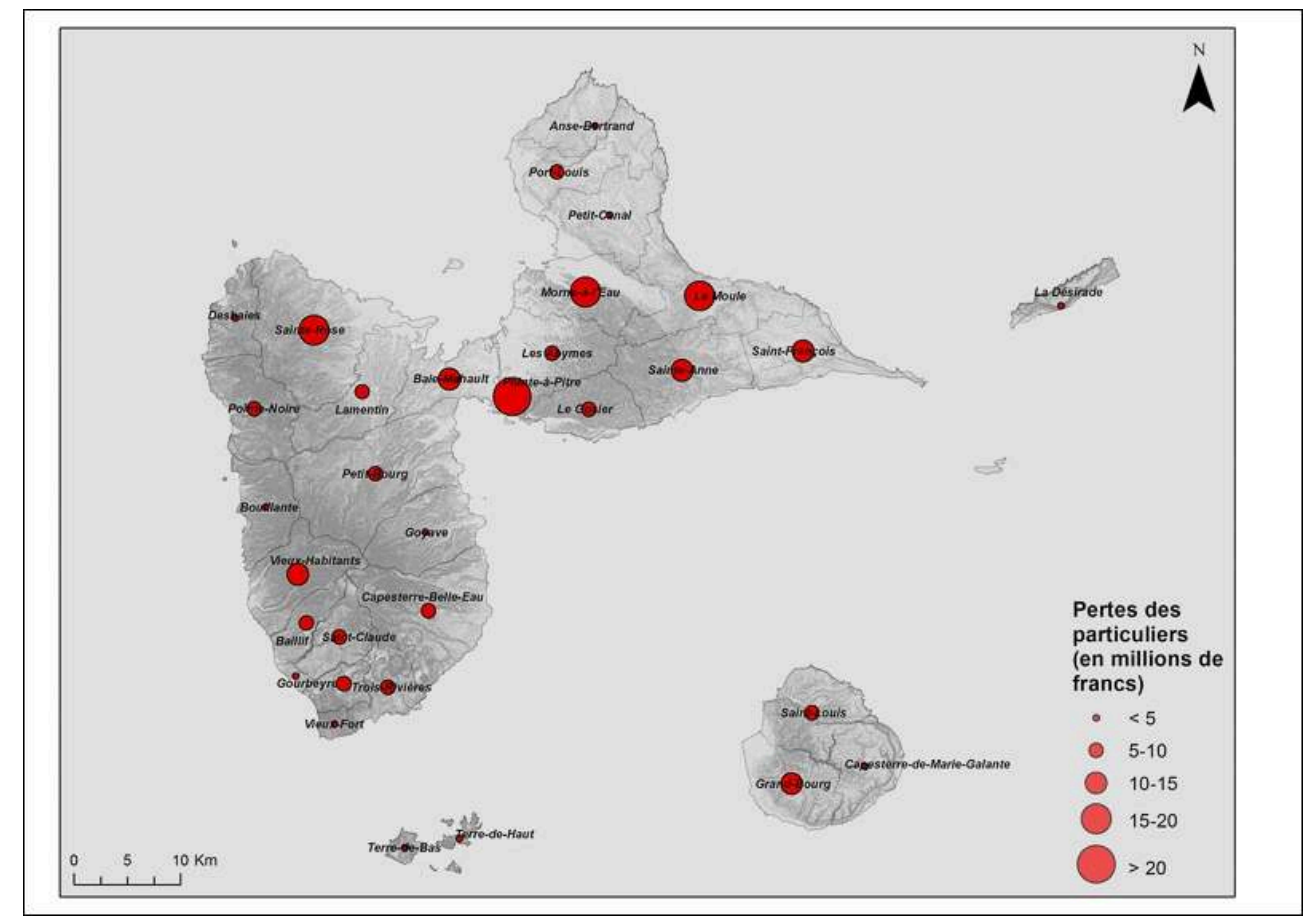

Source : ANOM, Guadeloupe, dossier 244, carton 1486

Les communes de la Grande-Terre regroupent $49 \%$ des dommages aux particuliers. Les communes de Pointe-à-Pitre, Sainte-Rose, Morne-À-L'eau et Le Moule sont les plus touchées et concentrent $34 \%$ des dommages. Ces niveaux de destruction s'expliquent par le fait que ces quatre communes regroupent $26 \%$ de la population de l'île à l'époque. Pointe-à-Pitre est la ville la plus peuplée de l'île avec plus de 26000 habitants en 1928. La population de Sainte-Rose, Morne-À-L'eau et Le Moule est respectivement d'environ 8000 habitants, 10000 habitants et 15000 habitants.

Les effets de site ont également pu jouer un rôle dans l'étendue des dommages. Comme l'a démontré l'analyse des dommages du cyclone Hugo en 1989, outre la position par rapport à la trajectoire de l'œil, intervient la situation par rapport aux dispositions du relief (Hamparian, 1999). Ainsi, l'exposition des communes peut être plurielle à l'image de Sainte-Rose où trois types de facteurs sont à prendre en compte. La première résulte de l'importance de la population sur la commune avec 8430 habitants en 1928 et du nombre de biens associés. Ensuite, la commune s'est trouvée sur la trajectoire du cyclone et enfin sa topographie avec un relief particulièrement maqué a accentué l'intensité de l'aléa.

\section{Les dommages aux activités}

$18 \mathrm{Au}$ moment de la survenance du cyclone, l'économie guadeloupéenne repose sur l'agriculture et plus particulièrement sur l'industrie sucrière avec 19 usines implantées sur l'île dont quatre à Marie-Galante (Touchelay, 2017). Si la culture de la canne à sucre prédomine, on trouve également le café, le cacao et la banane. Spatialement, la canne à 
sucre est omniprésente sur Grande-Terre tandis que les autres cultures se font sur Basse-Terre. Au lendemain du cyclone, toutes les infrastructures économiques sont à l'arrêt. Un grand nombre d'entre-elles nécessitent des travaux pour pouvoir redémarrer. Les déclarations des sinistrés pour l'obtention de secours permettent de mesurer l'impact sur les différentes activités économiques. Au total, 145 exploitants ou responsables d'entreprises se sont déclarés sinistrés (illustration 5).

Illustration 5 - Répartition des sinistrés par secteurs d'activités

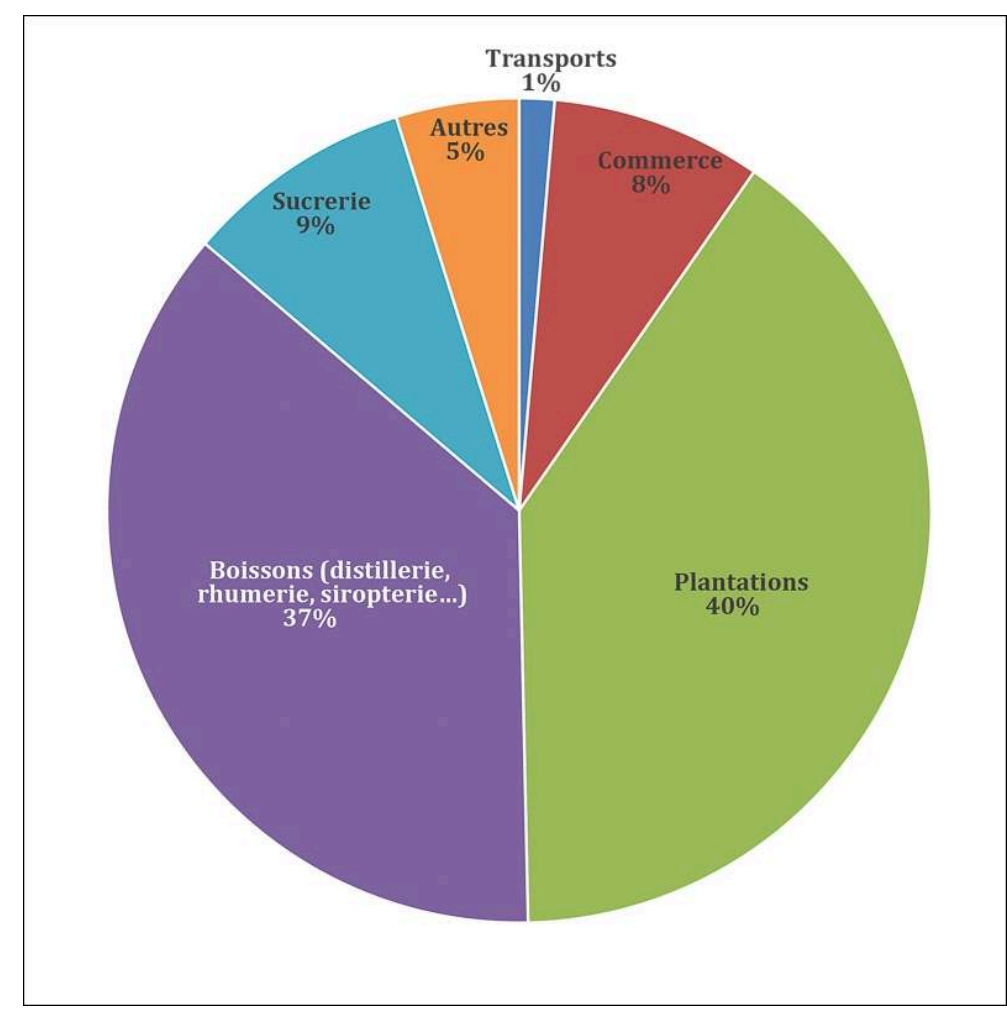

Source : AD Guadeloupe, série continue, carton 3).

19 Les différents planteurs comptent pour $40 \%$ des sinistrés. Cette forte représentation s'explique par les dommages importants aux cultures. En effet, $40 \%$ des plantations de cannes à sucre ont été détruites et environ $25 \%$ des cultures du café, du cacao et de la vanille n'ont pas survécu au passage du cyclone. À cela viennent s'ajouter la destruction de 9500 bananiers et de 7590 cocotiers. Les producteurs de boissons dont les infrastructures ont été endommagées pèsent pour $37 \%$ des sinistrés suivi des propriétaires de sucreries ( $9 \%$ ) et les commerçants $(8 \%)$.

$\mathrm{Au}$ total, les inventaires réalisés après le passage du cyclone estiment à environ 500 millions de francs à l'époque les dommages directs et indirects liés au passage du cyclone. Cette évaluation n'offre pas une vision fine de la nature des dommages sur les activités. L'analyse du montant des dommages issue des déclarations des sinistrés offre une autre représentation des conséquences de la catastrophe. Ainsi les 145 professionnels ont déclaré pour 68 millions de francs de dommages bien loin des 500 millions de l'évaluation globale qui prenait en compte le manque à gagner pour l'économie. Ainsi, l'activité liée à la culture de la canne à sucre, hors plantation, pèse pour $73 \%$ des dommages (illustration 6). Cette proportion s'explique par les lourdes infrastructures nécessaires à la filière sucrière et à la transformation de la canne. Ainsi 
le coût moyen d'un sinistre pour chacune des activités vient confirmer les lourdes conséquences du cyclone sur les infrastructures sucrières. Ainsi pour les sucreries, le coût moyen d'un sinistre est de 2,6 millions de francs. Pour la production de boissons, il est de 1,6 millions francs et de 190000 francs pour les plantations. Ces coûts moyens constituent des indicateurs importants pour saisir la vulnérabilité de la société guadeloupéenne. En effet, les coûts de reconstruction des usines de transformations ont eu des répercussions sur la reprise des activités.

Illustration 6 - Répartition des dommages déclarés par les sinistrés

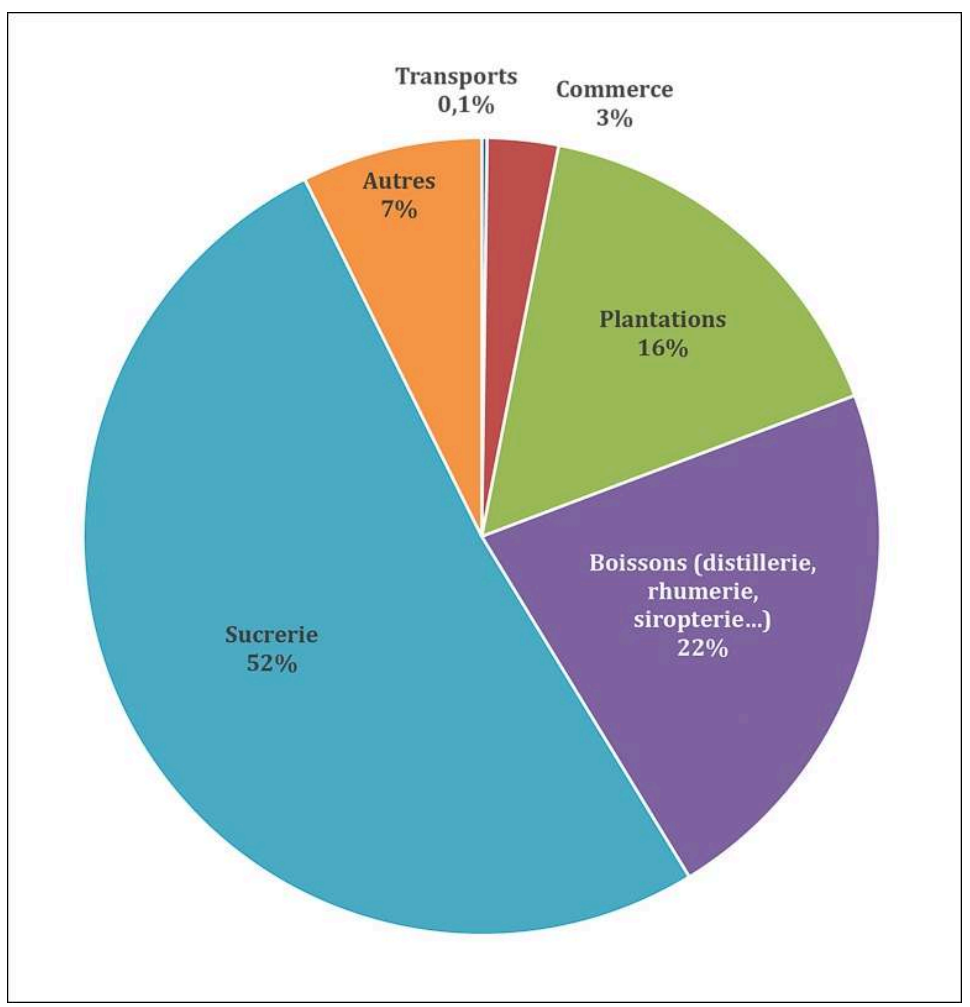

Source : AD Guadeloupe, série continue, carton 3.

21 La répartition spatiale des dommages permet d'appréhender l'exposition du territoire (illustration 7). Ainsi, avec un coût cumulé de 35 millions de francs, les communes de Pointe-à-Pitre, Sainte-Rose, Le Moule, Saint-François et Sainte-Anne concentrent $52 \%$ des dommages aux entreprises. Grande-Terre concentre $51 \%$ en raison principalement des dommages aux sucreries. Celles-ci comptent à elles seules pour 27 millions de francs sur les 35 millions de francs de dommages à Grande-Terre. 
Illustration 7 - Dommages déclarés aux entreprises en 1928

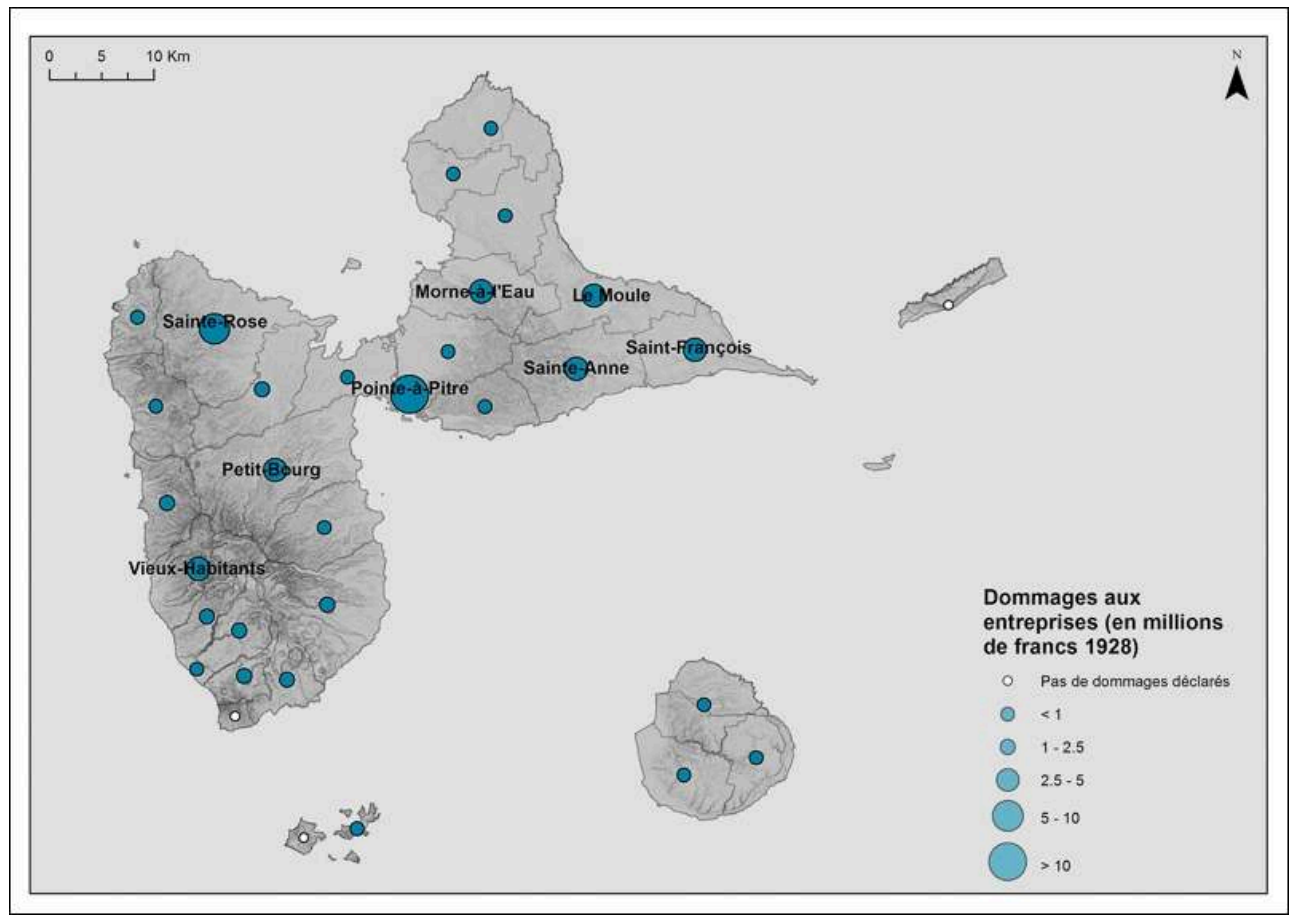

Source : AD Guadeloupe, série continue, carton 3

Aux dommages aux activités économiques s'ajoutent ceux des infrastructures communales (routes, ponts, édifices publics) pour environ 30 millions de francs. Leur destruction va peser sur les déplacements à l'intérieur de l'île, sur la reconstruction des bâtiments et sur la reprise des activités économiques. Au-delà de l'analyse des dommages directs, il convient également de s'interroger sur leurs répercussions sur l'économie de lîle.

\section{Quelles conséquences sur l'économie?}

Pour évaluer les conséquences de l'événement, les données statistiques sur les exportations disponibles dans l'annuaire statistique et des bulletins de l'Agence générale des colonies ont été mobilisées. Pour apprécier la situation économique, les données de la période 1924-1935 ont été retenues pour les quatre principales productions à savoir, le sucre, le rhum, le café et la banane (illustration 8). 
Illustration 8 - Exportations des principales cultures entre 1924 et 1935

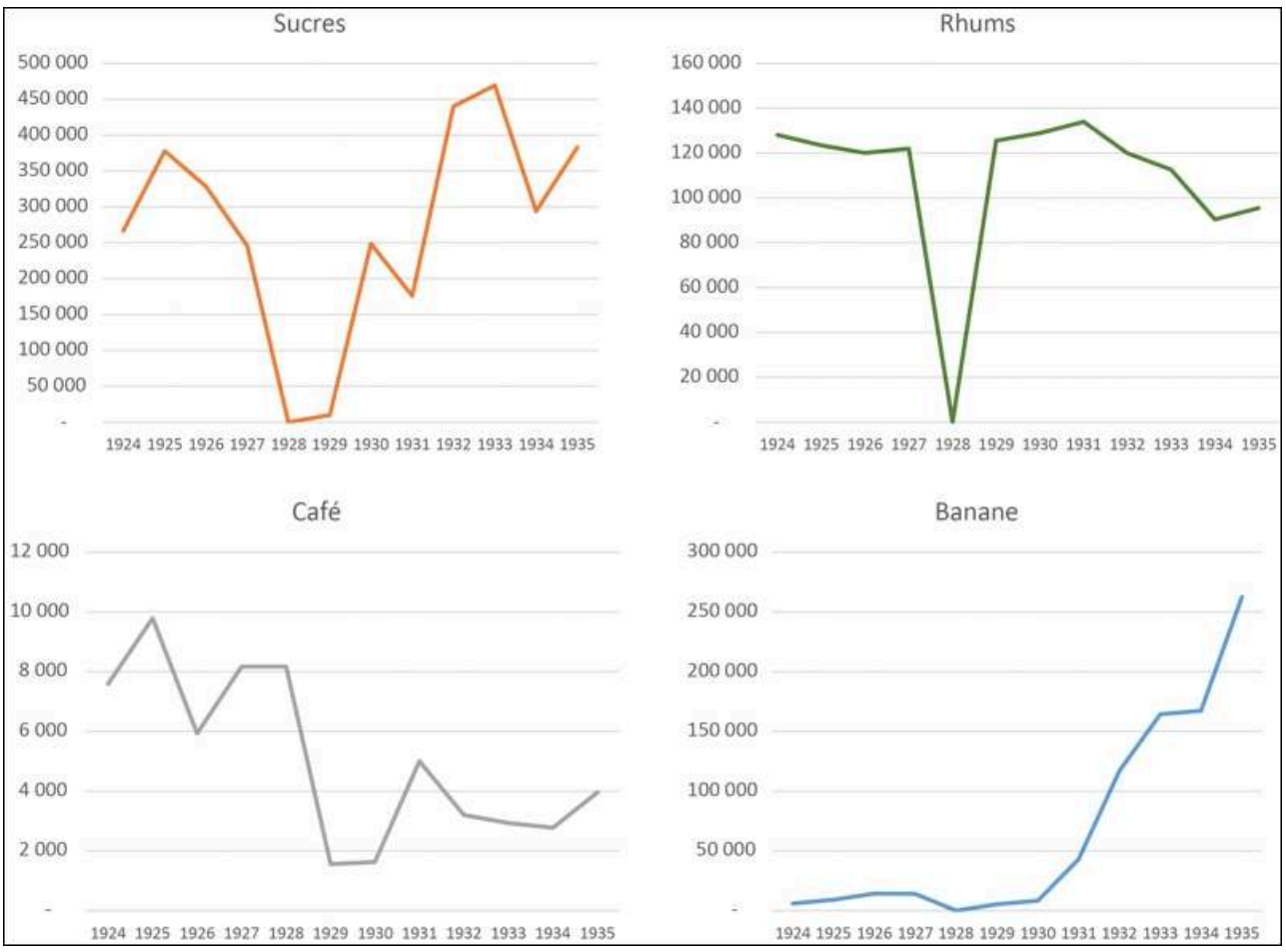

Sources: Annuaires statistiques de la France et Bulletins de l'agence générale des Colonies sur la période 1925-1936.

Les exportations de sucre connaissent une baisse en 1926 et 1927 après avoir atteint un pic en 1925. Avec le cyclone et la destruction des champs de cannes à sucre, les exportations atteignent leur plus bas niveau. L'année suivante, celles-ci redémarrent timidement mais il faut attendre 1930 pour que les exportations retrouvent leur niveau de 1927. La baisse des exportations en 1926 et 1927 est atténuée par la hausse des prix (Butel, 2007). Après une nouvelle baisse en 1931 liée à la sécheresse, les exportations atteignent les plus hauts niveaux de la période en 1933. Cependant, le prix du sucre voit son cours chuter en raison des effets de la crise économique de 1929 et de la concurrence du sucre de betterave. Ainsi, le quintal de sucre a perdu près de $20 \%$ de sa valeur entre la période 1924-1927 et la période 1930-1935.

Autre produit lié de la culture de la canne, le rhum connait également une baisse importante de ses exportations avec le passage de l'ouragan avant de retrouver dès 1929 un niveau similaire avant le passage du cyclone. Cette reprise rapide des exportations de rhum s'explique par les stocks constitués au cours des années précédentes et qui ont permis de pouvoir exporter d'importantes quantités de rhum dès l'année qui a suivi le passage du cyclone.

À l'inverse des deux précédents produits d'exportation, la culture du café connaît une autre trajectoire. En effet, si le cyclone n'a pas eu d'effet sur les exportations en 1928 puisque la récolte avait été effectuée avant le passage du cyclone, les années 1929 et 1930 connaissent leur plus bas niveau sur la période 1924-1935 avec moins de 2000 quintaux exportés annuellement. Malgré une reprise des exportations en 1931, le niveau ne reviendra jamais à ce qu'il était avant le passage du cyclone. Ceci s'explique par les mutations agricoles qui ont suivi le passage du cyclone de 1928. En raison du temps de croissance des plants, plusieurs producteurs décident d'abandonner le café au 
profit de la banane (Lafleur, 2006). En effet, la croissance des plants de café est relativement longue $-30 \mathrm{~cm}$ en dix mois - et la récolte devient abondante au bout de deux à trois années.

À l'opposé, le bananier présente l'avantage de produire des régimes au bout de seulement 10-12 mois. Avant le passage du cyclone, la culture de cette dernière est essentiellement une production de subsistance. Le gouverneur interdit son exportation à partir du 14 novembre 1928 jusqu'au $1^{\mathrm{er}}$ mars 1929 pour réserver la production aux seuls besoins des populations. À partir de 1931, les exportations de bananes connaissent une croissance annuelle importante. De moins de 50000 quintaux en 1931, les exportations dépassent les 250000 quintaux en 1935. Dans les années qui suivent le passage du cyclone, sa culture va être encouragée par les autorités pour contrebalancer les effets de la crise économique de 1929 sur le prix du sucre et du rhum (Cabre, 1929; Butel, 2007).

Sur l'ensemble des trois premiers trimestres 1929, les exportations s'élèvent à 119995975 francs soit un déficit de plus de 47 millions de francs par rapport à 1927. Dans le même temps, les importations s'élèvent à plus de 163 millions soit 56 millions de plus qu'en 1927 en raison de la fourniture de vivre et de matériaux nécessaires à la gestion de la catastrophe. Au total, l'analyse de l'ensemble des exportations et des importations permet d'appréhender les conséquences à moyen terme sur le commerce de la Guadeloupe (illustration 9).

Illustration 9 - Balance commerciale de la Guadeloupe entre 1924 et 1935 (en millions de francs)

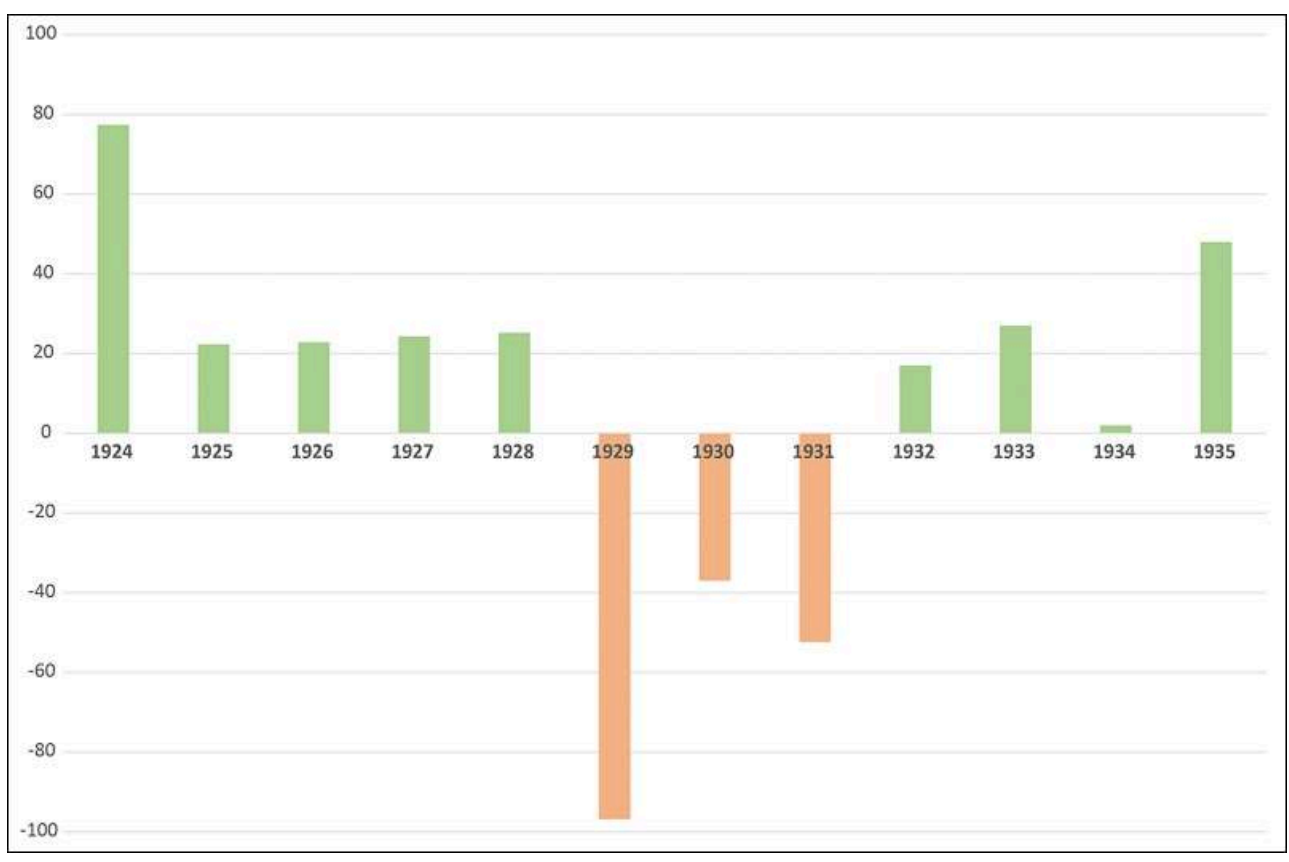

Sources: Annuaires statistiques de la France et Bulletins de l'agence générale des Colonies sur la période 1925-1936.

Entre 1924 et 1927, le montant des exportations est supérieur à celui des importations. De même pour 1928 qui malgré le passage du cyclone voit sa balance excédentaire grâce aux premiers trimestres de l'année. L'année 1929 accuse un déficit commercial total de près de 100 millions de francs en raison d'une baisse des exportations et d'une augmentation des importations liées à la reconstruction de l'île. L'économie 
guadeloupéenne retrouve son niveau antérieur à la catastrophe, quatre ans après sa survenance. Ce redressement de l'économie doit beaucoup aux dispositifs de secours mis en place par les autorités et dont il convient d'en analyser son organisation et son application.

\section{Les secours mis en œuvre pour faire face à la catastrophe}

\section{Les réponses apportées par l'État après la catastrophe}

Fidèle à ses habitudes, l'État intervient au lendemain de la catastrophe pour secourir les sinistrés et faire redémarrer l'économie au plus vite afin d'éviter que la catastrophe se mue en profonde crise économique et sociale (Favier, 2002; Desarthe, 2013). Les Antilles françaises de par leur importance dans l'économie française ont toujours bénéficié de secours au lendemain d'une catastrophe. Ainsi en 1865, 2 millions de francs avaient été mis à disposition des victimes de l'ouragan et de l'épidémie de choléra (ANOM, Guadeloupe, carton 117, dossier 816). Dans le cas de 1928, la gestion postcatastrophe se déroule dans un contexte de résurgence d'une rumeur de cession des Antilles françaises aux États-Unis en contrepartie de l'aide apportée pendant la Première guerre mondiale (Adélaïde-Merlande, 2014). Au lendemain du cyclone, les guadeloupéens vivent alors dans une terreur continuelle d'être cédée aux États-Unis (ANOM, Travaux Publics, carton 1157, dossier 2) et le gouvernement souhaite agir rapidement pour montrer le caractère indéfectible des liens qui unissent la Guadeloupe à la Métropole. De plus, les scènes de pillages et les émeutes qui ont marqué les jours qui ont suivi le passage du cyclone incitent le gouverneur à agir rapidement pour rassurer les populations.

31 Le gouverneur Tellier créé le 14 septembre 1928, trois commissions pour assurer la gestion post-catastrophe : une commission à l'échelle de chaque commune chargée de l'évaluation des dommages, une commission pour la centralisation des travaux et une commission pour la répartition des secours. Parallèlement, un service de comptabilité dédié au cyclone est mis en œuvre, alimenté au début par un fonds de 1,5 million de francs prélevé sur la caisse de réserve de la colonie et devant servir à assurer l'approvisionnement de l'île en vivres et médicaments.

De son côté, le ministère des colonies met en place une mission d'inspection confiée à l'inspecteur général des Colonies Muller pour suivre l'évaluation des dommages et la reconstruction. Le 26 septembre, le Parlement vote une subvention de 100 millions de francs pour venir en aide à la Guadeloupe. Cette somme représente $12,5 \%$ des dommages estimés et correspond à un peu plus de trois fois le budget de l'île. Sa gestion est confiée à une commission présidée par Michel Tardit membre de la section des finances, de la guerre, de la marine et des colonies au Conseil d'État. Elle a pour but d'arbitrer et de prioriser les dépenses.

À ce premier levier viennent s'ajouter deux dispositifs de prêts, un de 175 millions de francs avec le Crédit National et le second de 100 millions avec le Crédit Foncier devant permettre aux habitants de réparer ou reconstruire leurs habitations (ANOM, carton 244, dossier 1486). Enfin, 45 millions issus des indemnités de guerre dues par 
l'Allemagne et 4 millions de dons viennent compléter les ressources financières mais leur affectation n'est pas clairement définie.

Au total, 420 millions de francs sont mis à disposition des sinistrés du cyclone avec comme priorité affichée par le ministre des Colonies de restaurer d'une façon plus moderne l'outillage économique détruit (ANOM, Travaux Publics, carton 440, dossier 23).

\section{Une mise en œuvre difficile}

Dès son vote le 26 septembre 1928, la subvention de 100 millions de francs a été l'objet de dissensions politiques. Le libellé du décret attribuait par erreur cette somme au budget local de Guadeloupe. En conséquence, le Conseil Général demanda le versement de cette somme à son budget lui permettant ainsi d'avoir un droit de regard sur son application. Rapidement, plusieurs élus dont Gratien Candace se fait la voix militent pour cette solution. Cette situation illustre les forts enjeux politiques locaux de cette époque née à la fin de la Première Guerre Mondiale avec des revendications pour plus d'égalité sociale et une meilleure assimilation des citoyens antillais. Près de cinq mois après, le décret du gouverneur du 20 février 1929 vient clarifier la situation : le Conseil Général est exclu des discussions sur la répartition de la subvention. Pour asseoir sa décision, le gouverneur Tellier explique qu'un versement de la subvention au budget de la colonie aurait conduit à une annualisation de la subvention incompatible avec l'objectif d'un redressement rapide de l'île.

Ce décret fixe également la répartition de la subvention en différents postes de dépenses et permet d'appréhender les orientations faites par les autorités pour procéder au redressement de l'île (illustration 10). Ainsi, $14 \%$ de la subvention correspondent aux remboursements des frais engagés par le Gouverneur pendant la gestion de crise pour approvisionner l'île en vivres, médicaments et tôles nécessaires à la reconstruction. Les aides financières mobilisables sous la forme de prêts représentent $30 \%$ de la subvention. Un fonds de 16 millions de francs est prévu pour servir de garantie aux prêts des sinistrés. Une somme de 20 millions est réservée aux travaux publics pour la reconstruction des édifices publics (ponts, routes, bâtiments). Un fonds de réserve de 20 millions est mis en œuvre sans que son attribution soit clairement définie et qui sera finalement intégré aux prêts en 1931. 
Illustration 10 - Répartition de la subvention de 100 millions de francs votée par le Parlement

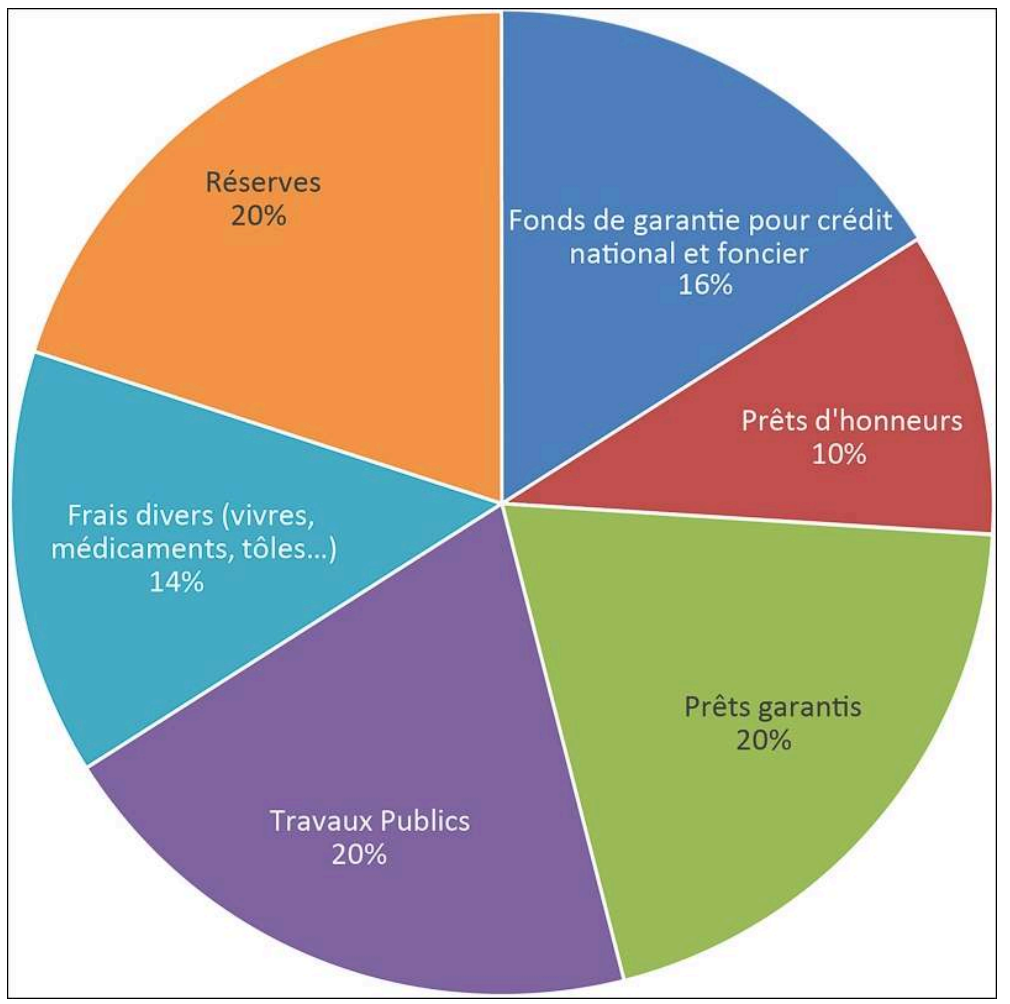

Source: ANOM, GUA, carton 244, dossier 1486.

37 En analysant les documents comptables nécessaires au suivi de la subvention conservés dans les archives, il est possible d'apprécier la mobilisation de cette subvention et d'avoir un aperçu sur l'avancée de la reconstruction de la Guadeloupe après le passage du cyclone jusqu'en 1934 où un état définitif est demandé par le ministre des Colonies (illustrations 11 et 12).

\section{Illustration 11 - Évolution de l'utilisation de la subvention votée par le Parlement}

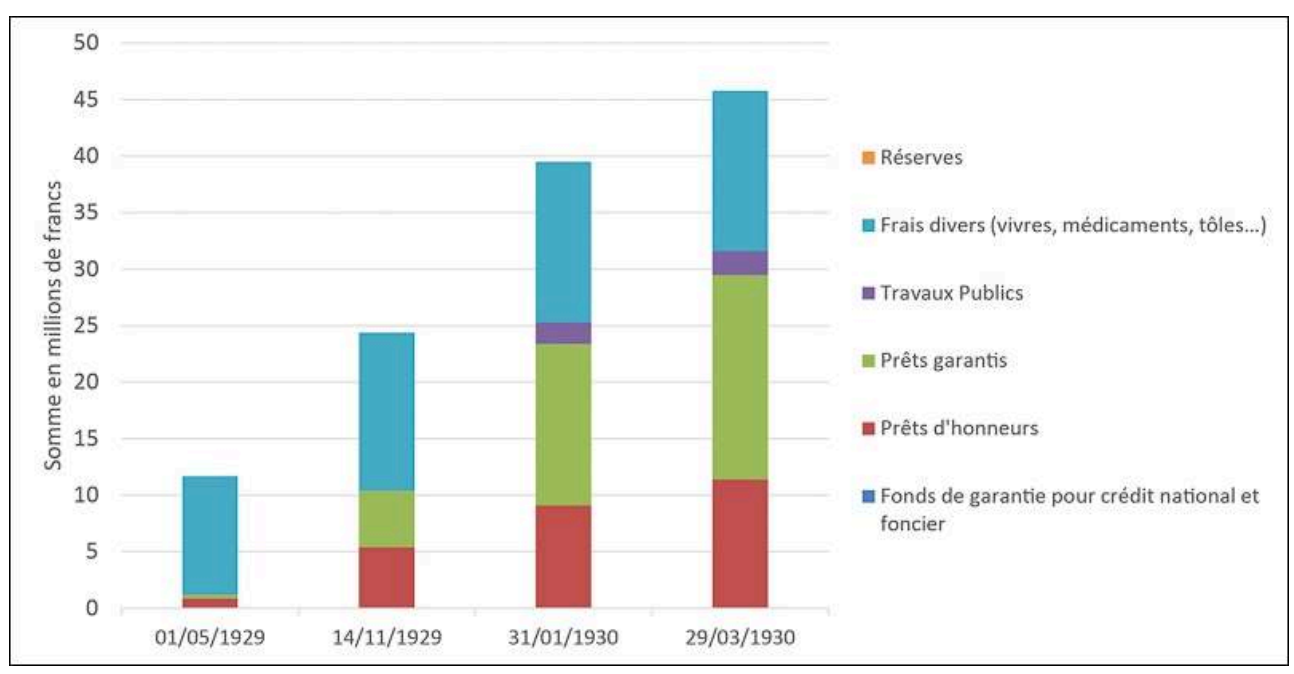

Source : ANOM, GUA, carton 244, dossier 1486. 
Regroupant, les dépenses réalisées dès le lendemain du cyclone, la tranche frais divers représente $90 \%$ des dépenses de la subvention au $1^{\mathrm{er}}$ mai 1929. Une analyse détaillée des dépenses relatives à cette tranche fait ressortir qu'elle a permis de financer l'achat de vivres, de médicaments et de tôles mais également la réparation de plusieurs édifices de la colonie et le rétablissement des réseaux de communications. Ainsi, dès le 27 octobre 1928, 28 kilomètres de routes ont été rénovées et le pont qui relie GrandeTerre et Basse-Terre est remis en fonction le mois suivant. À la fin du mois de novembre, 100000 tôles sont livrées pour reconstruire les toitures des habitations et des lieux publics.

Les oppositions politiques et les retards pris dans la répartition de la subvention ont repoussé la mise en place des prêts d'honneurs à $1 \%$ et des prêts garantis de $4 \%$ à avril 1929. Conformément au décret du 20 février 1929, les bénéficiaires sont les propriétaires d'usines et de plantation, illustrant ainsi la volonté de l'État de relancer en priorité les activités économiques. Au 15 septembre 1929, 4107 prêts ont été accordés et en novembre de la même année, 10,4 millions de francs sur les 30 millions de francs mobilisables ont été alloués. À la fin du mois de mars 1930, seulement 46 des 100 millions accordés ont été utilisés. Les fonds réservés aux prêts (prêts garantis et prêts d'honneurs) sont à $98 \%$ consommés. En revanche, les sommes consacrées aux travaux publics et au fonds de réserve n'ont pas encore été mobilisées.

Dix-huit mois après le passage du cyclone, la reconstruction de la Guadeloupe est loin d'être achevée. Les prêts en soutien à la filière sucrière ont permis de reconstituer une partie du tissu économique comme l'atteste la reprise des exportations de sucre observée plus haut. Dans un numéro de la revue des Annales coloniales de septembre 1929 consacrée à la Guadeloupe, un portrait idyllique de l'île est dressé par plusieurs personnes dont le sénateur Henry Béranger soulignant les qualités de l'île et son potentiel de développement économique (Béranger, 1929). Pour autant, derrière le tableau statistique flatteur présenté, plusieurs voix s'élèvent pour critiquer les choix fait dans l'aide aux sinistrés.

\section{Critiques et ajustements}

41 Au total, les 420 millions de francs mis à disposition ont permis de couvrir environ $53 \%$ des dommages. Dans un rapport daté du 15 mars 1929, l'inspecteur général des Colonies Muller estime que les moyens mis en œuvre sont adaptés aux besoins de l'île pour assurer sa reconstruction. Ainsi les prêts de 175 millions du Crédit National et celui de 100 millions du Crédit Foncier doivent couvrir la reconstruction ou la réparation des dommages privés qui rappelons-le s'élèvent à 250 millions de francs.

Cependant, seulement 70 millions ont été empruntés auprès du crédit national sur les 175 millions disponibles (ANOM, GUA, carton 244, dossier 1486). En fixant, le taux d'intérêt à $5 \%$ et dans le contexte d'un redémarrage difficile de l'économie synonyme de retour à l'emploi, une grande partie des sinistrés n'a pas été en mesure de faire un prêt. Les conditions nécessaires à l'obtention du prêt semblent avoir écarté beaucoup de sinistrés en raison de l'absence d'actes de propriétés exigées. Confronté aux critiques concernant l'octroi de prêt, le gouverneur déclare que quand il s'agit de l'argent du contribuable française on ne saurait parti de versements rapides, d'emploi immédiat car les synonymes de ces mots auraient été, en l'occurrence, gaspillage et prodigalités sans résultats (ANOM, GUA, carton 244, dossier 1486). 

l'industrie sucrière et a permis une restructuration rapide de la filière (Touchelay, 2017), les mesures dédiées aux populations sinistrées ne semblent pas avoir été pleinement satisfaisantes. Dans une lettre envoyée au ministre des Colonies au mois de mai 1929, le député Gratien Candace écrit «Depuis huit mois, les sinistrés de la Guadeloupe sont dans la plus noire des détresses et attendent désespérément les secours promis ou envoyé par la métropole [...] La population ouvrière des faubourgs vit dans des cahutes faites par ceux-là même qui les habitent, au milieu de flaques d'eau croupissantes. La filariose et le paludisme font des ravages parmi cette population " (ANOM, Guadeloupe, carton 244, dossier 1486). En défendant les intérêts de l'industrie sucrière, l'État a conforté la position sociale des familles qui ont la main sur les grandes entreprises. La question sociale si absente de la politique de secours jusqu'ici va rejaillir l'année suivante lors des grèves du mois de février 1930 à la suite d'une baisse des salaires des ouvriers agricoles (Butel, 2007).

Un an plus tard, le Parlement vote un nouveau crédit de 30 millions de francs afin de procéder à un rajustement définitif des mesures prévues en faveur des sinistrés de la Guadeloupe. Le 15 septembre 1931, une nouvelle répartition de la subvention est alors faite (tableau 1) (AD Guadeloupe, série continue, 3).

Tableau 1 - Évolution de la subvention et de sa répartition

\begin{tabular}{|l|c|c|}
\hline \multicolumn{1}{|c|}{ Mesures } & $\begin{array}{c}\text { Subvention de 100 } \\
\text { Millions de francs } \\
\mathbf{( 2 0 / 0 2 / 1 9 2 8 )}\end{array}$ & $\begin{array}{c}\text { Subvention de 130 } \\
\text { Millions de francs } \\
\mathbf{( 1 5 / 0 9 / 1 9 3 1 )}\end{array}$ \\
\hline $\begin{array}{l}\text { Fonds de garantie pour le crédit national } \\
\text { et le crédit foncier }\end{array}$ & 16 & 3 \\
\hline Prêts d'honneurs & 10 & 13 \\
\hline Prêts garantis & 20 & 40 \\
\hline Travaux Publics & 20 & 20 \\
\hline $\begin{array}{l}\text { Frais divers (vivres, médicaments, } \\
\text { tôles...) }\end{array}$ & 14 & 14 \\
\hline Réserves & 20 & 40 \\
\hline Allocation gratuite & $\mathbf{1 0 0}$ & $\mathbf{1 3 0}$ \\
\hline Total & & \\
\hline
\end{tabular}

Sources : ANOM, GUA, carton 244, dossier 1486 (pour la répartition de 100 millions francs) ; AD Guadeloupe, série continue, carton 3 (pour la répartition de 130 millions de francs).

Plusieurs mesures restent inchangées comme les frais divers et les travaux publics. En revanche, la capacité des prêts garantis passe de 20 à 40 millions de francs sous l'effet de l'intégration des fonds de la mesure Réserves. La baisse du fonds de garantie de 13 millions est répartie entre une hausse de 3 millions des prêts d'honneurs et les 10 millions restants sont ajoutés à la nouvelle subvention de 30 millions pour composer l'allocation gratuite de 40 millions.

Un rapport commandé par le ministre des Colonies daté du 25 juin 1934 permet d'avoir un aperçu de la situation des fonds utilisés six ans après le cyclone (illustration 12). Deux mesures ont vu leurs dépenses dépasser le plafond fixé. Il s'agit des dépenses diverses qui atteignent 17,1 millions en 1934 pour 14 millions prévus initialement, et 
des allocations gratuites, nées du réajustement de 1931 et dont le montant utilisé est supérieur de 1,1 million au plafond.

Illustration 12 - Utilisation de la subvention de 130 millions de francs au 25 juin 1934

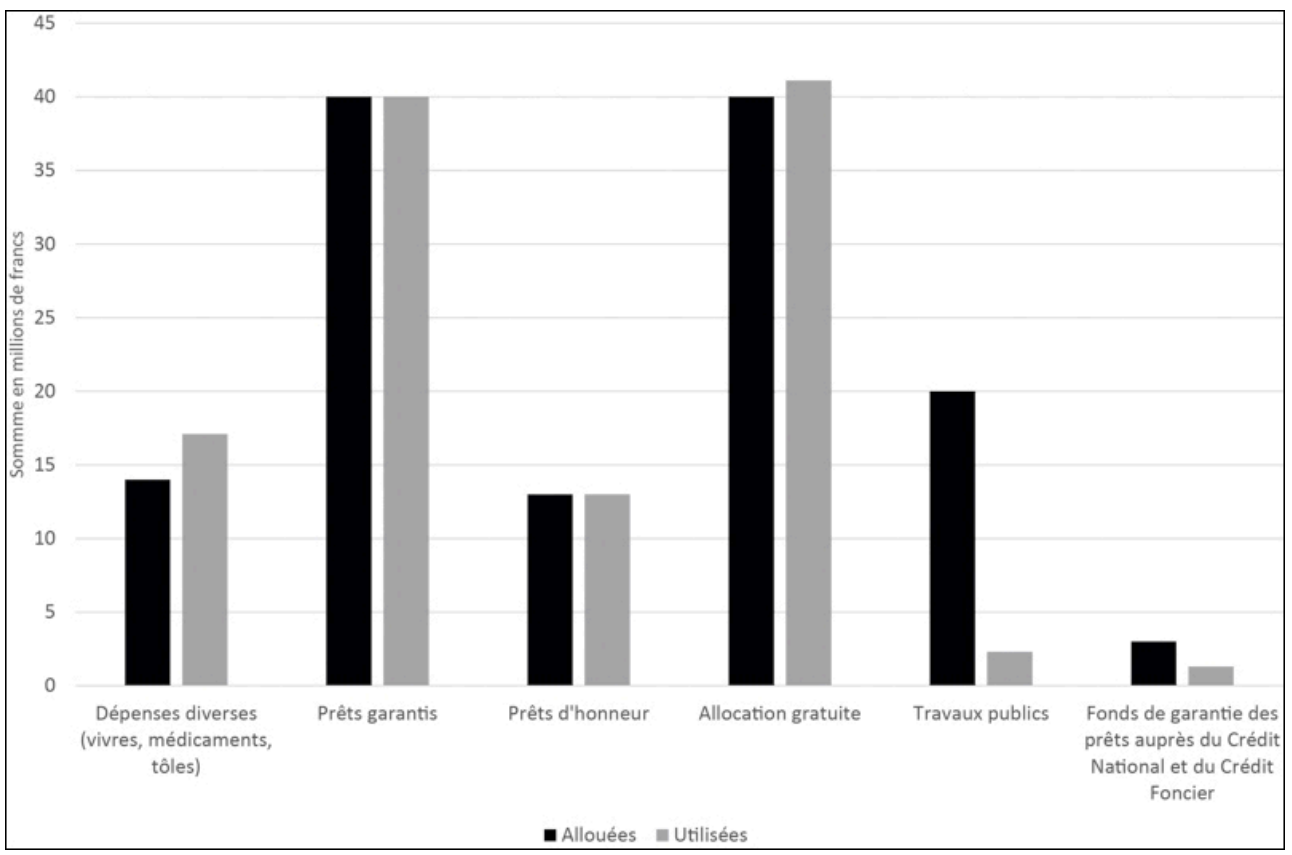

Source : AD Guadeloupe, série continue, carton 3.

En revanche, malgré le plan de reconstruction proposé par le gouverneur Tellier en 1929, le fonds de 20 millions de francs pour les travaux publics a été sous-utilisé puisque la dépense s'élève à 2,3 millions. Plusieurs raisons viennent expliquer le retard pris. Lors de l'estimation du montant des réparations, les prix de la reconstruction ont été décuplés par rapport au montant des dommages. En effet, plusieurs bâtiments publics détruits étaient en bois et leur remplacement en béton armé présente un surcoût (ANOM, TP, carton 440, dossier 23). Une dépêche ministérielle du 12 août 1929 est venu rappeler que la colonie doit être indemnisée en tant que victime du cyclone mais à ce titre seul. Ainsi les dépenses liées à l'amélioration du bâti pour une meilleure résilience ne sont pas éligibles dans le cadre de la subvention. À cela s'ajoute l'absence de main-d'œuvre qualifiée et de matériaux qui vont retarder la reconstruction des infrastructures de l'île. Pour cette raison, la mise en œuvre de constructions en béton armé sera plus longue que prévue mais se réalisera au cours des années 1930 sous la direction de l'architecte Ali Tur (Tur, 1930).

\section{Conclusion}

Le cyclone de 1928 constitue par son intensité un événement important de la mémoire des risques naturels de l'atlantique Nord. Pour la Guadeloupe, le cyclone se traduit par une mortalité jamais connu jusqu'ici (voir la contribution de Frédéric Leone et al. dans ce dossier). Survenu lors d'une période de faible activité cyclonique, l'ouragan est venu rappeler avec force la vulnérabilité des Antilles françaises. Il faudra ainsi attendre 1960 avec le passage de l'ouragan Donna sur Saint-Martin et Saint-Barthélemy pour voir un cyclone d'une telle intensité toucher de nouveau les Antilles françaises. 

l'origine de plusieurs grèves en février 1930 et rejailliront entre 1936 et 1938 (Buffon, 2006). En effet, en confortant la place de l'industrie sucrière, l'État a renforcé la domination des industriels sur les cultivateurs de cannes à sucre (Touchelay, 2017).

difficultés soulevées dans la gestion post-catastrophe ne sont pas sans rappeler celles rencontrées après le passage de l'ouragan Irma malgré les 80 années qui séparent les deux phénomènes. Malgré l'amélioration des systèmes de prévision et d'alerte, les territoires antillais conservent un caractère spécifique : leur insularité. Les ressources en main d'œuvre même limitée et malgré les difficultés d'approvisionnement de matériaux, le redémarrage des activités économiques peut être rapide. Toutefois, l'absence de politiques globales de gestion de la catastrophe intégrant les aspects sociaux est susceptible d'aggraver les disparités.

\section{BIBLIOGRAPHIE}

Annuaire Statistique de la France, 1925- 1935 (source imprimée).

Archives Nationales de l'Outre-Mer, Série Guadeloupe, carton 244, dossiers 1484, 1485, 1486, 1492.

Archives Nationales de l'Outre-Mer, Travaux publics, Guadeloupe, carton 1157, dossiers 2 et 3.

Archives Nationales de l'Outre-Mer, Travaux publics, Guadeloupe, carton 440, dossier 23.

Bulletins de l'Agence générale des colonies, 1925-1936 (source imprimée).

La Croix, 1928. Numéros du 18 septembre au 23 septembre.

L'Excelsior, 1928. Numéros du 17 septembre au 2 octobre.

Le Figaro, 1928. Numéros du 18 septembre au 23 septembre.

Adélaïde-Merlande J., 2014. Au lendemain de la Première Guerre Mondiale, va-t-on céder les Antilles françaises aux Etats-Unis d'Amérique ? Bulletin de la Société d'Histoire de la Guadeloupe, $n^{\circ} 168$, p. 199-202.

Béranger H., 1929. L'île d'émeraude : Notre belle Guadeloupe. Les Annales coloniales. Revue mensuelle illustrée, septembre 1929, p. 1-5. DOI: https://doi.org/10.7202/1026852ar

EchoGéo, 51 | 2020 
Butel P., 2007. Histoire des Antilles françaises. Paris, Perrin, coll. Tempus, 566 p.

Buffon A., 2006. Eboué et les grèves en Guadeloupe (1936-1938). Bulletin de la Société d'Histoire de la Guadeloupe [En ligne], n¹43-144, p. 45-87. DOI: https://doi.org/10.7202/1040686ar

Cabre A., 1929. Production et commerce de la banane en Guadeloupe, Les Annales coloniales. Revue mensuelle illustrée, septembre 1929, p. 6-8.

Chathuant D., 2003. Gratien Candace : une figure de la vie politique française. $1^{\text {ère }}$ partie : la IIIe République (1900-1940), Bulletin de la Société d'Histoire de la Guadeloupe [En ligne], n¹34, p. 27-106. DOI: https://doi.org/10.7202/1040745ar

Cœur D., 2006. La plaine de Grenoble face aux inondations. Genèse d'une politique publique du XVII au $\mathrm{XX}$ siècle. Paris, QUAE, $310 \mathrm{p}$.

Dauphine A., Provitolo D., 2007. La résilience : un concept pour la gestion des risques. Annales de Géographie, n 654, p. 115-125.

Desarthe J., 2013. Le temps des saisons : climat, événements extrêmes et sociétés dans l'Ouest de la France (XVIe-XIXe siècle). Paris, France, Hermann, 338 p.

Desarthe J., 2015. Ouragans et submersions dans les Antilles françaises (XVII ${ }^{\mathrm{e}}-\mathrm{XX}^{\mathrm{e}}$ siècle), Études caribéennes [En ligne], $\mathrm{n}^{\circ}$ 29. DOI: https://doi.org/10.4000/etudescaribeennes.7176

Desarthe J., Moncoulon D., 2017. Quatre siècles de cyclones tropicaux dans les départements français d'outre-mer. La Météorologie [En ligne], nº 99. DOI: https://doi.org/10.4267/2042/63590

Desarthe J., 2019. Les RETEX dans l'histoire : une pratique ancienne de gestion des risques. In Rey $\mathrm{T}$, Defossez S., Retours d'expériences post-catastrophes naturelles. Actes du colloque Géorisques, $\mathrm{n}^{\circ} 8$, p. $19-30$

Duvat V., 2008. Le système du risque à Saint-Martin (Petites Antilles françaises), Développement durable et territoires. Économie, géographie, politique, droit, sociologie [En ligne], dossier 11. DOI: https://doi.org/10.4000/developpementdurable.7303

Duvat V., Magnan A., Canavesio R., 2017. La reconstruction des chaînes d'impacts au service de l'évaluation de la résilience des territoires et de la réduction des risques météo-marins : le cas des atolls des Tuamotu, Polynésie française. Colloque Risques et résilience des territoires : apports de la notion de résilience à la gestion des risques, SHF, Novembre 2017, Marne La Vallée. URL : https:// hal.archives-ouvertes.fr/hal-01634349

Duvergé P., 1995. Le Service météorologique colonial. La Météorologie, NS, p. 46-51.

Fabre F., Stehle G., 1992. Le cyclone de 1928 à la Pointe-à-Pitre. Bulletin de la Société d'Histoire de la Guadeloupe[En ligne], n91-94. DOI: https://doi.org/10.7202/1043731ar

Farge A., Welfelé O., 2002. Penser et définir l'événement en histoire. Terrain. Revue d'ethnologie de l'Europe, $\mathrm{n}^{\circ}$ 38, p. 67-78.

Favier R., Granet-Abisset A-M., 2000. Histoire et mémoire des risques naturels. Grenoble, MSH-Alpes, $281 \mathrm{p}$.

Favier R., Granet-Abisset A-M., 2002. Les pouvoirs publics face aux risques naturels dans l'histoire. Grenoble, MSH-Alpes, $444 \mathrm{p}$.

Fassig O., 1928. San Felipe. The hurricane of september 13, 1928 at San Juan. Monthly Weather Review, vol. 9, $\mathrm{n}^{\circ}$ 56, p. 350-352.

Garnier E., 2009. Les dérangements du temps. 500 ans de chaud et de froid en Europe. Paris, Plon, 245 p. 
Giaconna F., Martin B., Eckert N., Desarthe J., 2019. Une méthodologie de la modélisation en géohistoire : de la chronologie (spatialisée) des événements au fonctionnement du système par la mise en correspondance spatiale et temporelle. Physio-Géo, vol. 14, p. 171-199.

Hamparian R., 1999. L'ouragan Hugo sur la Guadeloupe : les enseignements d'une cartographie des effets locaux. In Les Antilles, terres à risques, Paris, Karthala, p. 19-39.

Lafleur G., 2006. La culture du café en Guadeloupe, de son introduction à sa quasi-disparition. Bulletin de la Société d'Histoire de la Guadeloupe [En ligne], n¹45. DOI: https://doi.org/ 10.7202/1040672ar

Le Gouvernement de Martinique, 1937. Le service météorologique et de physique du Globe de la Martinique (Antilles françaises). Paris, Larose, $48 \mathrm{p}$.

Léone F., Vinet F., 2006. La vulnérabilité des sociétés et des territoires face aux menaces naturelles. Analyses géographiques. Montpellier, Presses Universitaires de la Méditerranée, 140 p.

Le Roy Ladurie E., 1967. Histoire du climat depuis l'an mil. Paris, Flammarion, 376 p.

Meschinet De Richemond N., 2016. Modernité, anachronisme et ambivalence des risques et catastrophes naturelles à travers l'approche géohistorique. Vertigo [En ligne], vol. 16, $\mathrm{n}^{\circ}$ 3. DOI: https://doi.org/10.4000/vertigo.18034

Mitchell C., 1928. The west indian hurricane of September 10-20, 1928. Monthly Weather Review, vol. 56, n 9, p. 347-350.

Provitolo D., 2005. Un exemple d'effets de domino : la panique dans les catastrophes urbaines. Cybergeo [En ligne], document 328. DOI: https://doi.org/10.4000/cybergeo.2991

Saffache P., Marc, J-V, Huyghes-Belrose V., 2003. Les cyclones en Gudeloupe. Quatre siècles cataclysmiques. Ibis-Rouge, $276 \mathrm{p}$.

Schnakenbourg C., 2000. La création des usines en Guadeloupe (1843 - 1884). Recherche sur la modernisation de l'industrie sucrière antillaise après l'abolition de l'esclavage. Bulletin de la Société d'Histoire de la Guadeloupe [En ligne], n 124-125. DOI: https://doi.org/10.7202/1043188ar

Schnakenbourg C., 2009. L'industrie sucrière en Guadeloupe aux XIX $X^{e}$ et XX $X^{e}$ siècle. Paris, L'Harmatan, $242 \mathrm{p}$.

Touchelay M-C., 2017. La Guadeloupe, une île entreprise, des années 1930 aux années 1960 : les entrepreneurs, le territoire, l'État. Thèse de doctorat, Université Sorbonne-Paris, $610 \mathrm{p}$.

Tur A., 1930. Notes sur la reconstruction de la Guadeloupe. Basse-Terre, Imprimerie Catholique, 247 p.

Vallette C., Cartier S., 2012. Dénombrer pour maîtriser les dommages des catastrophes naturelles. Vertigo [En ligne], vol. 12, nº 1. DOI: https://doi.org/10.4000/vertigo.12138

Veyret Y., 2007. Dictionnaire de l'environnement. Paris, Armand Colin, 404 p.

Walker B., Holling C.S., Carpenter S.R., Kinzig A., 2004. Resilience, Adaptability and Transformability in Social-ecological Systems. Ecology and Society [En ligne], vol. 9, n². URL: http://www.ecologyandsociety.org/vol9/iss2/art5/

Zahibo N., Pelinosky E., Talipova T., et al., 2007. Statistical analysis of cyclone hazard for Guadeloupe, Lesser Antilles. Atmospheric research [En ligne], vol. 84, n 1, p. 13-29. DOI: https:// doi.org/10.1016/j.atmosres.2006.03.008 


\section{RÉSUMÉS}

L'ouragan de septembre 1928 qui a frappé les Antilles et plus particulièrement la Guadeloupe constitue une des catastrophes naturelles les plus importantes qu'a connue ce territoire au cours $\mathrm{du} \mathrm{XX}^{\mathrm{e}}$ siècle. L'exploitation des archives administratives disponibles rend possible l'analyse des conséquences de l'événement à travers les dommages inventoriés et les secours mis en œuvre. Ce faisant, il est possible d'appréhender les différentes temporalités de la catastrophe jusqu'en 1934.

The hurricane of September 1928 which struck the Antilles and especially the Guadeloupe constitutes one of the most important natural disasters that experienced this territory during the XXth century. From the use of the available archives, it is possible to analyze the consequences of the event through the damage inventoried and the assistance implemented. So, it is possible to apprehend the different temporality of the disaster until 1934.

\section{INDEX}

Keywords : hurricane, history of catastrophe, vulnerability, resilience, Guadeloupe Mots-clés : ouragan, histoire des catastrophes, vulnérabilités, résilience, Guadeloupe Thèmes : Sur le Champ - Sur le Terrain

\section{AUTEUR}

\section{JÉRÉMY DESARTHE}

Jérémy Desarthe, jdesarthe@ccr.fr, est chargé de mission prévention des risques naturels à la Caisse Centrale de Réassurance, Paris. Il a récemment publié :

- Desarthe J., 2019. Les RETEX dans l'histoire : une pratique ancienne. In Rey T., Defossez S., Retours d'expériences post-catastrophes naturelles. Presses Universitaires de la Méditerranée, p. 19-30.

- Giacona F., Martin B., Eckert N. Desarthe J., 2019. Une méthodologie de la modélisation en géohistoire : de la chronologie (spatialisée) des événements au fonctionnement du système par la mise en correspondance spatiale et temporelle. Physio-Géo [En ligne], vol. 14. DOI: https:// doi.org/10.4000/physio-geo.9186

- Metzger A., David F., Valette P. et al., 2018. Entretenir la mémoire des inondations via les repères de crue ? Développement Durable \& Territoires [En ligne], vol. 9, $\mathrm{n}^{\circ}$ 3. DOI: https://doi.org/ 10.4000/developpementdurable.12937 